\title{
Évaluation des coûts associés aux troubles anxieux et aux troubles de l'humeur au moyen d'une enquête téléphonique
}

\author{
SB Patten, M.D., Ph.D. (1); JVA Williams, M.Sc. (1); C Mitton, Ph.D. (2)
}

\section{Résumé}

Les études de coûts sont essentielles aux décisions stratégiques en matière de santé. Les estimations de coûts disponibles concernant les troubles anxieux et les troubles de l'humeur au Canada ne sont, cependant, peut-être plus à jour. Dans la présente étude, nous avons estimé un ensemble de coûts directs liés aux soins de santé, en utilisant les données recueillies au moyen d'une enquête téléphonique provinciale portant sur les troubles anxieux et les troubles de l'humeur en Alberta. L'enquête a fait appel à la méthode de composition aléatoire afin de joindre un échantillon de 3394 résidants de ménages âgés de 18 à 64 ans. L'entrevue téléphonique comprenait des questions permettant d'évaluer les coûts, sans indiquer si ces coûts étaient engagés par le répondant, le gouvernement ou un régime de santé. L'entrevue intégrait également la mini-entrevue diagnostique neuropsychiatrique (Mini Neuropsychiatric Diagnostic Interview [MINI]). Les coûts associés aux antidépresseurs semblent avoir augmenté depuis la publication des dernières estimations de coûts disponibles. Étonnamment, la majeure partie des coûts des antidépresseurs étaient engagés par les répondants ne présentant aucun trouble décelé. De plus, contre toute attente, une proportion importante des coûts des médicaments se rapportaient aux médicaments psychotropes autres que les antidépresseurs et les anxiolytiques-sédatifs-hypnotiques. De tels résultats laissent croire que les coûts associés au traitement antidépresseur ont subi des changements notables. Il est possible que les données disponibles sur les coûts de la maladie ne soient plus à jour et que certaines hypothèses émises dans des études précédentes ne soient plus valables aujourd'hui.

Mots clés : études transversales, troubles dépressifs, coûts et analyse des coûts

\section{Contexte}

Les troubles dépressifs constituent un défi important pour la santé de la population ${ }^{1}$. Selon le projet sur le fardeau mondial de la maladie, la dépression majeure unipolaire est le quatrième principal facteur en cause dans le fardeau de la maladie à l'échelle mondiale; elle occupe le second rang dans les pays développés comme le Canada² Les répercussions de ce trouble sur la santé de la population tiennent, en partie, à sa prévalence élevée : environ $5 \%$ des Canadiens connaissent un épisode de dépression majeure au cours d'une année donnée ${ }^{3}$. Les répercussions sont toutefois accrues par le fait que la prévalence culmine dans le groupe d'âge de 25 à $44 \mathrm{ans}^{4}$, ce qui correspond à une période cruciale sur le plan de la formation scolaire, de l'établissement de relations et de la productivité économique. La dépression majeure est également un trouble récurrent, et elle est fréquemment associée à une comorbidité psychiatrique et médicale ${ }^{5,6}$. Tous ces facteurs ont tendance à accroître les effets de ce trouble sur la santé de la population. Finalement, la dépression majeure a une incidence sur la mortalité7.

Diverses études de coûts sur les troubles dépressifs ont été réalisées à l'échelle inter- nationale. Ces études avaient habituellement pour objectif d'estimer les coûts globaux, incluant les coûts directs et indirects liés aux traitements. Les études de coûts en population utilisent habituellement des données provenant de diverses sources, telles que les rapports gouvernementaux, les données administratives et les enquêtes nationales. Une telle approche s'explique par le fait que les données recueillies auprès d'une source particulière (p. ex. une enquête nationale) ne sont probablement pas suffisamment exhaustives pour permettre un calcul détaillé des coûts.

Récemment, Thomas et Morris ${ }^{8}$ ont publié des estimations des coûts liés à la dépression en Angleterre. Leur étude intègre des données provenant d'un grand nombre de sources et fournit les estimations les plus justes à ce jour pour l'Europe ${ }^{9}$. Cependant, l'approche adoptée pour évaluer certains des éléments de coûts était peu rigoureuse. Ainsi, dans l'évaluation des coûts liés aux médicaments, on a estimé la prévalence de la dépression (cas traités) au moyen d'une formule utilisant comme point de départ des données administratives relatives aux consultations chez le médecin. Le nombre estimatif de personnes ayant consulté un médecin pour une dépression était multiplié par la proportion d'antidépresseurs prescrits à l'échelle nationale selon des catégories précises d'antidépresseurs, puis par les coûts moyens des ordonnances dans ces catégories. Une telle approche suppose que le coût total correspondant à la "prévalence traitée » multipliée par le coût par personne traitée. L'approche semble raisonnable a priori,

\footnotetext{
Coordonnées des auteurs
}

1 Université de Calgary, Département des sciences de la santé communautaire, Calgary (Alberta)

2 Études sur la santé, Université de la Colombie-Britannique, Kelowna (Colombie-Britannique)

Correspondance : Scott B. Patten, Université de Calgary, Département des sciences de la santé communautaire, 3330 Hospital Drive NW, Calgary (Alberta),

Tél. : 403-220-8752, Courriel : patten@ucalgary.ca 
mais elle ne tient pas compte du fait que le traitement antidépresseur est souvent poursuivi longtemps après la rémission des épisodes de dépressifs ${ }^{10}$.

Stephens et Joubert ${ }^{11}$ ont estimé le fardeau total de la dépression majeure et de la détresse au Canada en 1998, en utilisant également des données provenant de diverses sources. Leur estimation des coûts totaux s'élevait à 14,4 milliards de dollars. Leur analyse était fondée, d'une part, sur les données de l'Enquête nationale sur la santé de la population (ENSP) et, d'autre part, sur les données recueillies dans le cadre du projet « Le fardeau économique de la maladie au Canada (FEMC) », 1998 (http://www.phac-aspc.gc.ca/publicat/ ebic-femc98/index_f.html). Toutes les données sur les coûts des médicaments provenaient du rapport FEMC. De leur côté, les estimations du FEMC reposaient sur une base de données de suivi des coûts de l'Institut canadien d'information sur la santé (ICIS), laquelle contient les estimations des coûts effectuées par une société privée : IMS Health Canada. Une telle approche est pertinente parce que les bases de données d'IMS Health Canada permettent de déterminer le volume total des ventes de médicaments et la proportion de recommandations de traitement pour des indications particulières. Selon le FEMC, 108 millions de dollars ont été dépensés en médicaments pour les états anxieux et 252 millions de dollars, pour les troubles dépressifs. Toutefois, il y a déjà 10 ans que cette analyse a été réalisée, et certaines de ces estimations ont été faites il y a presque 15 ans. La période qui a suivi a été marquée par la reconnaissance accrue du fait que la dépression majeure est un problème de santé, ce qui a sans doute pour effet de réduire le caractère honteux associé à ce trouble; on a aussi assisté à l'émergence de nouveaux traitements pharmaco-logiques et non pharmacologiques.

En 2003, une enquête provinciale sur la santé mentale (Alberta Mental Health Survey), parrainée par l'Alberta Mental Health Board, a été réalisée en Alberta ${ }^{12}$. Une autre initiative (l'Alberta Depression Initiative), a été mise sur pied en 2004 par
Santé et Bien-être Alberta, et gérée par l'Institut d'économie de la santé. Dans le cadre de cette deuxième initiative, une seconde enquête téléphonique provinciale axée sur la santé mentale a été réalisée au cours de l'année suivante; cette fois, l'enquête mettait l'accent sur la dépression. Certains résultats de l'enquête, qui sont résumés dans ce rapport, sont pertinents pour les prochaines études de coûts sur les troubles anxieux ou les troubles de l'humeur au Canada.

\section{Méthodologie}

\section{Collecte des données de l'enquête}

L'Alberta est une province canadienne qui compte 3,3 millions d'habitants répartis sur un territoire de $661190 \mathrm{~km}^{2}$. Les méthodes d'enquête téléphonique constituaient la stratégie la plus réaliste pour obtenir un échantillon presque représentatif de cette population dispersée sur le plan géographique. Environ les deux tiers de la population albertaine résident dans deux villes : Edmonton et Calgary. Le plan d'échantillonnage était stratifié, de façon que le tiers de l'échantillon provienne de chacune de ces villes, et le reste, des régions rurales. La collecte des données s'est limitée aux résidants de ménages, âgés de 18 à 64 ans, ayant une ligne téléphonique résidentielle.

En 2005, la collecte des données a été effectuée par l'unité d'enquête sur la population du service Quality, Safety and Health Information [QIHI]) de la région sanitaire de Calgary (www.calgaryhealth region.ca). Au départ, on a sélectionné un échantillon de numéros de téléphone résidentiels dans la province, et le dernier numéro a été remplacé par un nombre aléatoire afin d'inclure les numéros n'apparaissant pas sur la liste ${ }^{13,14}$ et d'éviter d'introduire un biais dans l'éventualité où les ménages ayant un numéro non inscrit présenteraient des caractéristiques différentes de ceux ayant un numéro inscrit. Pour chaque ménage joint, la personne à interroger était sélectionnée au hasard au moyen d'une technique pseudo-aléatoire, la " méthode du dernier anniversaire de naissance ». Jusqu'à neuf tentatives de rappel ont été faites dans le but de joindre toutes les personnes échantillonnées. Ces appels ont été effectués durant les heures de travail, le soir et la fin de semaine.

La mini-entrevue diagnostique neuropsychiatrique (MINI), une courte entrevue diagnostique structurée $e^{15,16}$, a été utilisée comme indicateur diagnostique de la dépression majeure et d'un ensemble d'autres troubles anxieux et troubles de l'humeur courants. Dans le cas des épisodes de dépression majeure, l'évaluation a porté sur la prévalence au cours des 14 derniers jours (essentiellement, la prévalence instantanée pour ce trouble); quant à la dysthymie, l'évaluation a reposé sur la prévalence au cours des deux dernières années. En ce qui concerne le trouble panique, l'agoraphobie, le trouble d'anxiété généralisée et la phobie sociale, la prévalence actuelle a aussi été évaluée. Puisqu'il a été établi que les différences entre les instruments d'enquête sont souvent attribuables au rôle des indicateurs de la « signification clinique » dans les algorithmes de $\operatorname{cotation}^{17}$, nous avons intégré une question relative à la perturbation, en demandant aux répondants si leurs symptômes psychiatriques perturbaient leur vie. Les épisodes étaient considérés comme cliniquement significatifs, si les répondants faisaient état " de nombreuses » perturbations.

Un module axé sur la pharmacoépidémiologie et un autre visant à recenser les traitements non pharmacologiques ont aussi été inclus dans l'entrevue. Le module pharmaco-épidémiologique était élaboré selon une structure cyclique : on se renseignait d'abord sur les médicaments pris pour le traitement de symptômes sommairement définis mais pertinents (p. ex. «Prenez-vous actuellement des médicaments de prescription pour l'anxiété, la dépression, le stress, le niveau d'énergie, le sommeil, le soulagement de la douleur, la fibromyalgie ou la migraine? »), puis on revenait sur chaque médicament déclaré, en posant une série de questions sur le nombre de comprimés et la taille des comprimés, les raisons justifiant la prise des médicaments et la durée de l'utilisation. On incitait les répondants à déclarer 
l'information figurant sur les étiquettes de leurs flacons de comprimés pour garantir l'exactitude de cette information. La posologie a été établie en combinant l'information sur la taille des comprimés ou capsules pertinents pris et sur leur nombre, incluant les schémas de prescription "au besoin » (prn). Les doses ont été consignées en milligrammes par jour. L'entrevue comprenait également des questions évaluant la fréquence d'utilisation des services de santé. Ces questions étaient habituellement jumelées, c.-à-d. qu'un premier élément portait sur l'utilisation d'un service et le second, sur la fréquence d'utilisation. Voici un exemple de question : "Au cours des 6 derniers mois, avez-vous consulté un spécialiste autre qu'un omnipraticien, un médecin de famille, un urgentologue ou un psychiatre au sujet de votre état de santé? » Si la réponse était « oui », la question suivante était : "Combien de fois? ». Nous avons eu recours à des questions semblables pour évaluer les consultations auprès de médecins de famille, de psychiatres, de psychologues, de travailleurs sociaux ainsi que les consultations aux urgences et aux cliniques sans rendezvous, les examens radiologiques et les hospitalisations.

\section{Méthode de calcul des coûts}

Nous avons obtenu les coûts par milligramme pour chaque médicament figurant dans le guide de calcul des coûts du régime provincial d'assurance-médicaments (http:// www.pao.gov.ab.ca/benefits/choice/ prescription-drug-plan.pdf). Lorsque les coûts par milligramme différaient selon la forme posologique, nous avons calculé, pour chaque médicament, le coût pour la plus faible dose en milligrammes. Les coûts associés aux préparations génériques ont été utilisés lorsqu'une telle préparation était disponible, puisque les pharmaciens peuvent remettre un produit de remplacement à moindre coût lorsqu'ils exécutent une ordonnance. Lorsqu'une personne prenait plus d'un médicament d'une classe particulière, les coûts étaient estimés de la même façon pour chaque médicament, puis les coûts de chaque médicament étaient additionnés. Pour faciliter l'interprétation des données, nous avons multiplié les coûts quotidiens par 365, afin d'estimer le coût annuel des médicaments. Une telle approche semble raisonnable puisque $80 \%$ des répondants à l'enquête qui ont déclaré prendre des antidépresseurs ont aussi mentionné qu'ils prenaient ces médicaments pendant plus d'un an. La même méthode a été utilisée pour les anxiolytiques-sédatifs-hypnotiques et les antipsychotiques.

En ce qui a trait à l'utilisation des services professionnels, les codes d'honoraires associés aux divers groupes professionnels ont été utilisés. Dans le cas des médecins, les codes d'honoraires du régime d'assurancemaladie de l'Alberta ont été utilisés. Pour les médecins de famille, nous avons utilisé un code de base pour une consultation au cabinet ne nécessitant pas une anamnèse et une évaluation complètes (code d'honoraires 03.03A). Les consultations aux urgences ont été traitées distinctement, en utilisant un coût estimatif par consultation correspondant au double des honoraires pour une consultation auprès d'un médecin de famille. Pour les spécialistes, nous nous sommes servis du code d'honoraires pour une consultation de 15 minutes recommandée par un médecin généraliste (code d'honoraires 03.03FA). Les honoraires associés à une prise en charge clinique de 45 minutes ont été utilisés pour les psychiatres (code de niveau de compétence $08.19 \mathrm{~A}$ ), tandis que, pour les psychologues, le barème d'honoraires publié par l'association provinciale des psychologues a été utilisé.

Nous n'avons pas été en mesure de détailler les examens radiologiques et analyses de laboratoire déclarés par les participants à l'enquête. Nous avons plutôt dégagé des estimations de coûts médianes représentatives pour les examens courants ou habituels dans chaque catégorie. Dans cette étude, ces examens étaient les suivants : radiographie pulmonaire, tomodensitométrie de base de la tête et IRM de base de la tête. Les estimations des coûts par examen diagnosti-que utilisées dans l'étude sont tirées d'un guide de calcul des coûts élaboré par la région sanitaire de Calgary. Ce guide a pour but d'assurer le recouvrement des coûts des projets de recherche, incluant les coûts du matériel et des fournitures et ceux associés au personnel. Parallèlement, pour établir les coûts moyens quotidiens associés aux hospitalisations dans l'ensemble de la province, nous nous sommes servis des estimations faites par la région sanitaire de Calgary à des fins administratives. Les documents élaborés par la région sanitaire de Calgary ont été considérés comme une source acceptable, puisque la région comprend non seulement la ville de Calgary, mais également les régions rurales s'étendant au sud-ouest de la ville jusqu'à la frontière de la Colombie-Britannique.

Pour chaque catégorie, nous avons effectué une projection des coûts pour la population totale. La prévalence pondérée pour la catégorie diagnostique trouble de l'humeur et/ou trouble anxieux a été multipliée par la population provinciale totale dans la plage d'âge étudiée, la proportion de personnes recourant à un examen, traitement ou service particulier ainsi que les coûts moyens afférents afin de produire une estimation finale des coûts. Les poids d'échantillonnage ont été calculés comme étant l'inverse de la probabilité de sélection estimée dans les catégories d'âge et de sexe de chacune des trois régions d'échantillonnage pour la population provinciale. Le poids obtenu a, de nouveau, été corrigé en le multipliant par le ratio du nombre de résidants admissibles dans chaque ménage divisé par le nombre de lignes téléphoniques de chaque ménage. Les poids ont été traités comme des poids relatifs durant l'analyse.

\section{Résultats}

Au total, 18113 numéros de téléphone ont été composés. Plus de la moitié d'entre eux ont été exclus de l'échantillon pour les raisons suivantes : dans 1663 ménages, il n’y avait pas de résidants admissibles; 329 appels ont été bloqués; 846 appels ont abouti à un répondeur ou à une boîte vocale (c.-à-d. aucune personne n'a pu être jointe, malgré les rappels); 1747 numéros correspondaient à des lignes commerciales; 3333 numéros n'étaient pas en service; 1385 numéros correspondaient à des numéros de télécopieur; 378 appels ont joint des ménages non anglophones; 845 appels n'ont jamais obtenu de réponse au cours des protocoles de rappel; 11 appels ont été interrompus de manière hostile et 79 numéros ont été 
jugés inadmissibles pour diverses raisons. Finalement, 7497 appels ont réussi à joindre des ménages admissibles. On a enregistré 3443 refus $(45,9 \%)$ au niveau des ménages. Parmi les 4054 ménages dans lesquels des répondants pouvaient être choisis, 635 refus individuels ont été notés $(15,7 \%)$. Nous avons été en mesure de mener l'entrevue au complet avec presque tous les 3419 répondants consentants, à l'exception de 25 d'entre eux $(0,7 \%)$, de sorte que 3394 entrevues ont été effectuées. Après des vérifications de l'exactitude et de l'intégralité des données, 45 dossiers ont été retirés de l'ensemble de données en raison de préoccupations relatives à la qualité des données. L’analyse finale portait sur les données recueillies auprès des 3345 personnes restantes $(82,5 \%$ des sujets qui ont été invités à participer et qui ont fourni des données adéquates). Toutefois, si l'on calcule le taux de réponse en utilisant le nombre de ménages admissibles comme dénominateur, il se situe à 44,6\%. En utilisant la méthode de calcul de l'Association de la recherche et de l'intelligence marketing (ARIM) (http://www.tpsgc-pwgsc.gc.ca/ por/text/pebptel-intro-f.html), 7628 des 18113 répondants peuvent être classés comme étant " hors du champ d'enquête ", ce qui porte à $31,9 \%$ le taux de réponse.

Nous avons dénombré 168 participants à l'enquête ayant un trouble de l'humeur (dépression majeure ou dysthymie actuelle) selon la MINI, ce qui a permis d'estimer la prévalence à 4,6 \%. Cette estimation concorde avec la littérature existante. La prévalence de la dépression majeure sur une période de 30 jours dans l'Enquête sur la santé dans les collectivités canadiennes : santé mentale et bien-être (ESCC 1.2) se situait à 1,8 \% ${ }^{4}$. L'ESCC 1.2 n'a pas évalué le trouble dysthymique, mais Waraich, dans une recension systématique, a estimé que la prévalence au cours de l'année précédente était d'environ $2 \%{ }^{18}$. Cent quatre-vingtdouze répondants présentaient un ou plusieurs troubles anxieux, ce qui porte à $6,3 \%$ la prévalence estimative. Ce chiffre est légèrement plus élevé que l'estimation de la prévalence du trouble anxieux au cours de l'année précédente $(4,7 \%)$ fournie par l'ESCC $1.2^{19}$, mais cette enquête n'a pas évalué le trouble d'anxiété généralisée, dont la prévalence annuelle dans la population atteint 3,1 \% aux États-Unis ${ }^{20}$. Chez la plupart des répondants atteints de troubles, le trouble anxieux et le trouble de l'humeur étaient souvent concomitants. Seulement $2,2 \%$ des répondants étaient atteints uniquement d'un trouble anxieux ou d'un trouble de l'humeur, tandis que 4,1\% des répondants présentaient un trouble anxieux et un trouble de l'humeur concomitants. Étant donné que la MINI constitue un bref protocole de dépistage, sa capacité de différencier le trouble anxieux du trouble de l'humeur est douteuse. L'analyse a, par conséquent, été axée sur deux groupes : les répondants atteints d'un trouble anxieux ou d'un trouble de l'humeur (mais non les deux) et les répondants présentant un trouble anxieux et un trouble de l'humeur concomitants.

En ce qui concerne la prise de médicaments au moment de l'enquête, 7,4 \% de la population a déclaré prendre un antidépresseur, 3,1 \%, un sédatif hypnotique et $1,5 \%$, un autre médicament possiblement relié au traitement des troubles de l'humeur : des psychorégulateurs, des psychostimulants ou des antipsychotiques atypiques. Bien que les médicaments de ce dernier groupe soient distincts sur le plan pharmacologique, ils ont été regroupés dans l'analyse sub-séquente parce qu'ils n'étaient pas suffisam-ment nombreux pour qu'il soit possible de les examiner séparément. Il faut également noter que le libellé d'introduction des questions indiquait que les médicaments d'intérêt étaient ceux pris à des fins psychotropes, et que cette catégorie n'inclurait pas, par exemple, les psychorégulateurs anticonvulsivants pris pour le traitement de l'épilepsie.

Des 2,2\% de la population présentant un trouble anxieux ou un trouble de l'humeur non concomitants, 20,4\% prenaient un ou plusieurs antidépresseurs. Parmi les répondants atteints d'un trouble anxieux et d'un trouble de l'humeur concomitants, 44,4\% prenaient un ou plusieurs antidépresseurs. Dans le groupe ne présentant aucun trouble anxieux ou trouble de l'humeur décelé, 5,6 \% prenaient un antidépresseur. Le coût annuel moyen estimé des antidépresseurs dans chacun de ces groupes est résumé dans le tableau 1. Au moment de l'enquête, on dénombrait 2105167 résidants âgés de 18 à 64 ans dans la province. Le coût moyen par personne multiplié par la prévalence estimée et le nombre de personnes dans la province pour chaque catégorie diagnostique a mené à une estimation du coût total de 124 millions de dollars pour les antidépresseurs. Ce chiffre incluait sept millions de dollars pour les sujets présentant un trouble anxieux ou un trouble de l'humeur non concomitant, 35 millions de dollars pour les sujets présentant des troubles concomitants et 83 millions de dollars pour les sujets ne présentant aucun trouble décelé par la MINI.

Bien que la fréquence à laquelle les répondants ne présentant aucun trouble anxieux ou trouble de l'humeur décelé prenaient des antidépresseurs était relativement faible (5,6 \%) et que le coût annuel prévu par personne dans ce groupe était légèrement inférieur à celui établi dans le groupe atteint de troubles décelés par la MINI (voir tableau 1), le coût total dans ce groupe était plus élevé parce que le nombre de personnes appartenant à cette catégorie était supérieur. Un tel résultat laisse croire que la plupart des coûts associés au traitement antidépresseur sont maintenant engagés durant les phases de poursuite et d'entretien du traitement ${ }^{10}$, puisque ces répondants ne satisfont plus aux critères symptomatiques des troubles anxieux ou des troubles de l'humeur. Le résultat n'est pas tellement surprenant, puisque le traitement aigu par les antidépresseurs dure habituellement 6 à 8 semaines, tandis qu'il est recommandé, en règle générale, de prescrire le traitement d'entretien durant un an ou plus, et un traitement pendant une durée indéfinie peut être indiqué chez les sujets atteints de troubles très récurrents ${ }^{10}$. D’autres facteurs pourraient toutefois expliquer un tel résultat, comme nous le verrons ci-dessous.

Un profil semblable a été observé dans le cas des sédatifs hypnotiques (voir tableau 1). Chez les personnes souffrant de troubles anxieux ou de troubles de l'humeur, la fréquence de la prise de ces médicaments était beaucoup plus élevée que chez les répondants ne présentant pas de tels troubles. Cependant, la plupart des 
sujets prenant ces médicaments ne présentaient pas un trouble anxieux ou un trouble de l'humeur. Par conséquent, la majeure partie des coûts était reliée au groupe non atteint d'un trouble. Le coût total estimatif associé à la prise d'un sédatif hypnotique dans la population était de 8 millions de dollars, ce qui est considérablement inférieur au coût des antidépresseurs.

Divers autres médicaments ont été déclarés par les répondants à l'enquête : le lithium $(\mathrm{n}=7)$, la carbamazépine $(\mathrm{n}=3)$, le valproate $(n=5)$, la gabapentine $(n=9)$, la lamotrigine $(n=1)$, le topiramide $(n=20)$, la dexamphétamine $(\mathrm{n}=6)$, la chlorpromazine $(\mathrm{n}=1)$, l'olanzapine $(\mathrm{n}=10)$, la quétiapine $(\mathrm{n}=8)$, la clozapine $(\mathrm{n}=2)$ et le rispéridone $(n=20)$. Quatre-vingt-douze répondants ont déclaré prendre un ou plusieurs de ces médicaments. Malheureusement, seuls 69 de ces répondants (75\%) étaient disposés ou en mesure de fournir de l'information sur la posologie. Considérée comme un groupe, une proportion estimée à $1,5 \%$ de la population totale prenait au moins un médicament appartenant à cette catégorie. La fréquence de la prise chez les répondants présentant un trouble anxieux ou un trouble de l'humeur non concomitants était semblable à celle de la population totale (voir tableau 1), mais la fréquence était beaucoup plus élevée dans le groupe dont les troubles étaient concomitants $(16,4 \%)$. Environ 1 \% de la population non atteinte d'un trouble anxieux ou d'un trouble de l'humeur prenait l'un de ces médicaments - résultat presque concordant avec la prévalence des troubles psychotiques dans la population. Le coût total prévu était de 38 millions de dollars; de ce montant, 25 millions de dollars étaient attribuables au groupe ne présentant pas de trouble décelé. Cependant, ces résultats laissent croire qu'une proportion assez importante des coûts de traitement pour les troubles anxieux et les troubles de l'humeur ne seraient désormais plus liés aux classes pharmaceutiques que l'on a toujours considérées comme étant les plus importantes dans le traitement des troubles anxieux et des troubles del'humeur : les antidépresseurs et les sédatifs hypnotiques.

Le tableau 2 présente les coûts associés à l'utilisation des services offerts par les professionnels de la santé mentale. La fréquence à laquelle les répondants ont eu recours aux services des psychiatres et des psychologues était considérablement supérieure chez les personnes atteintes de troubles anxieux et de troubles de l'humeur, particulièrement dans la catégorie de comorbidité. Chez les sujets atteints d'un trouble anxieux ou d'un trouble de l'humeur, ces coûts ont triplé, tandis que dans le groupe atteint de troubles concomitants, les coûts ont augmenté par un facteur de huit. Cependant, la majeure partie des coûts associés aux psychologues relèvent du groupe ne présentant pas de trouble défini par la MINI, ce qui laisse croire que ces professionnels exercent souvent des activités thérapeutiques qui ne sont pas expressément axées sur ces troubles, p. ex. la psychothérapie conjugale. Le tableau 3 présente les coûts associés à l'utilisation des services offerts par des médecins de famille et des spécialistes autres que les psychiatres. Les répondants atteints de troubles anxieux et de troubles de l'humeur étaient plus nombreux à consulter un médecin, et ce, à une plus grande fréquence. Ces deux facteurs sont

TABLEAU 1

Coûts annuels et semestriels associés à la prise de médicaments, selon la catégorie de troubles

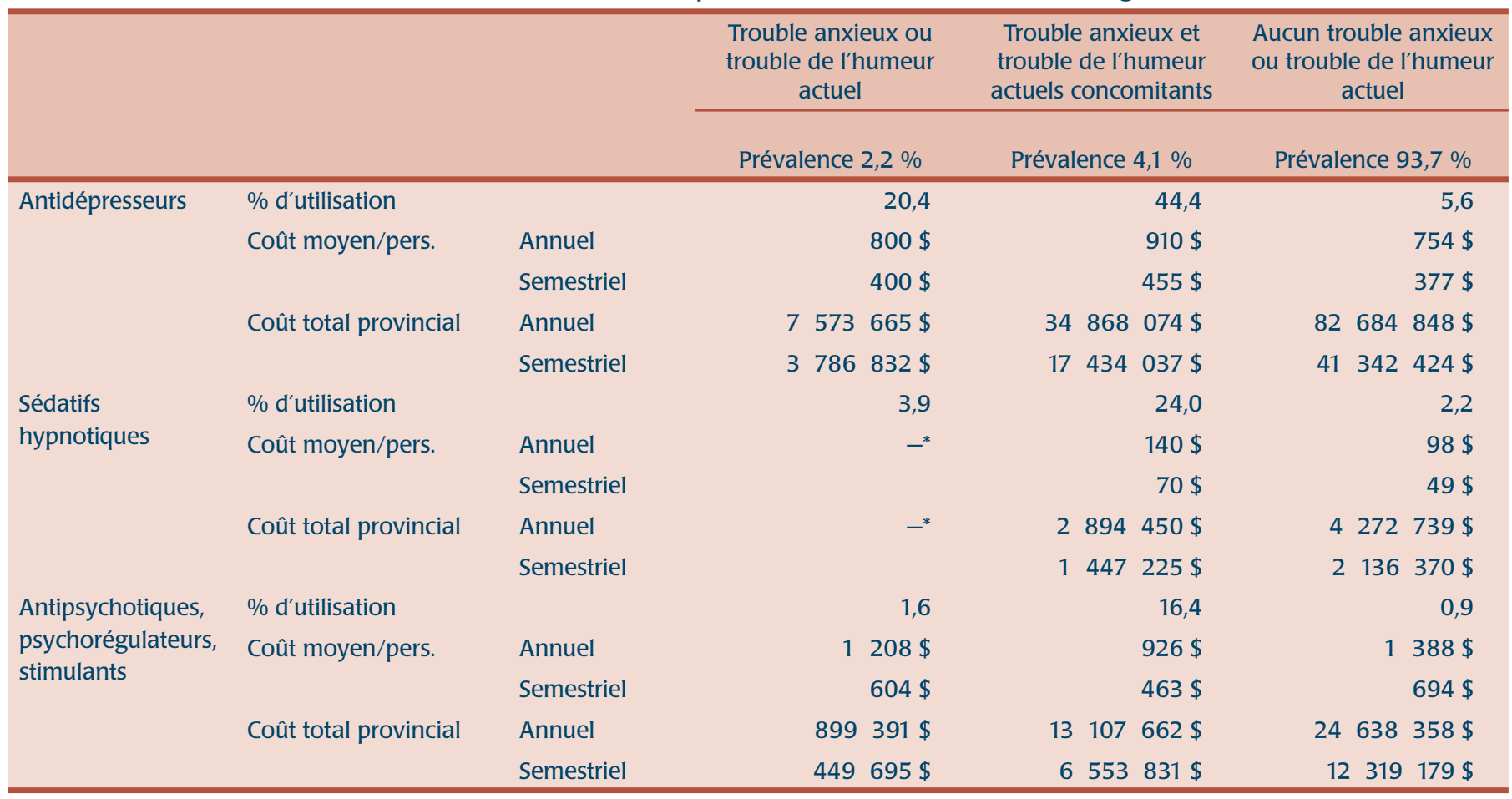

${ }^{*}$ Nombres trop faibles pour étayer l'estimation. 
TABLEAU 2

Utilisation des services offerts par les spécialistes de la santé mentale, selon la catégorie de troubles (durant les 6 mois précédant l'enquête)

\begin{tabular}{|c|c|c|c|c|c|c|c|}
\hline \multirow[b]{2}{*}{ Psychiatre } & & \multicolumn{2}{|c|}{$\begin{array}{l}\text { Trouble anxieux ou trouble } \\
\text { de l'humeur actuel }\end{array}$} & \multicolumn{2}{|c|}{$\begin{array}{c}\text { Trouble anxieux et trouble } \\
\text { de I'humeur actuels } \\
\text { concomitants }\end{array}$} & \multicolumn{2}{|c|}{$\begin{array}{c}\text { Aucun trouble anxieux ou } \\
\text { trouble de I'humeur } \\
\text { actuel }\end{array}$} \\
\hline & $\%$ d'utilisation & & 6,1 & & 26,1 & & 1,8 \\
\hline & Nombre moyen de consultations & & 2,4 & & 10,4 & & 3,2 \\
\hline & Coût total provincial & 996 & $754 \$$ & 33811 & $137 \$$ & 16544 & $730 \$$ \\
\hline \multirow[t]{4}{*}{ Psychologue } & $\%$ d'utilisation & & 6,2 & & 14,5 & & 1,7 \\
\hline & Nombre moyen de consultations & & 4,7 & & 6,6 & & 4,2 \\
\hline & Coût moyen/pers. & & $698 \$$ & & $987 \$$ & & $632 \$$ \\
\hline & Coût total provincial & 2013 & $150 \$$ & 12354 & $147 \$$ & 21110 & $090 \$$ \\
\hline
\end{tabular}

responsables des coûts plus élevés par atteints de troubles anxieux et de troubles répondant dans le groupe souffrant de de l'humeur concomitants.

troubles anxieux et de troubles de l'humeur.

Les données relatives au calcul des coûts des examens diagnostiques sont présentées dans le tableau 4. Dans le cas des examens radiologiques, la fréquence des examens (et, par conséquent, les coûts par sujet selon les examens utilisés dans cette étude) ne différait pas selon la catégorie de troubles. Cependant, la proportion de sujets dans les divers sous-groupes ayant passé de tels examens était supérieure chez les sujets atteints de troubles anxieux et de troubles de l'humeur. Comme l'indique le tableau 5, les coûts associés aux consultations aux urgences et aux hospitalisations étaient également élevés chez les répondants

\section{Conclusions}

Bon nombre des résultats présentés dans le présent rapport concordent avec la littérature existante sur les études de coûts associés aux troubles dépressifs. Ils mettent en évidence l'augmentation des coûts associés à la prise de médicaments et à l'utilisation des services chez les personnes atteintes de ces troubles. Comme on s'y attendait, cette observation est valable à la fois pour les mesures des soins de santé mentale et les soins de santé généraux. Une question importante qui se pose sur le plan de l'interprétation est le fait que les troubles de l'humeur sont étroitement associés aux troubles médicaux chroniques ${ }^{21-23}$, de sorte que ces coûts élevés peuvent refléter un effetindirect des troubles non psychiatriques. Sachant qu'environ $10 \%$ de la population canadienne vit en Alberta, les résultats laissent entendre qu'il y a eu une augmentation notable des coûts associés à la pharmacothérapie pour traiter la dépression, depuis la publication du FEMC, 1998. Dans le rapport FEMC, les coûts totaux à l'échelle nationale associés aux médicaments contre les troubles anxieux et les troubles de l'humeur étaient seulement deux fois plus élevés que ceux observés dans la province de l'Alberta seulement. En d'autres mots, si l'on suppose que $10 \%$ des 360 millions de dollars estimés dans le FEMC ont été dépensés en Alberta, le coût estimatif pour

TABLEAU 3

Coûts associés à l'utilisation des services offerts par les médecins de famille et les spécialistes (excluant les psychiatres) selon la catégorie de troubles (durant les 6 mois précédant l'enquête)

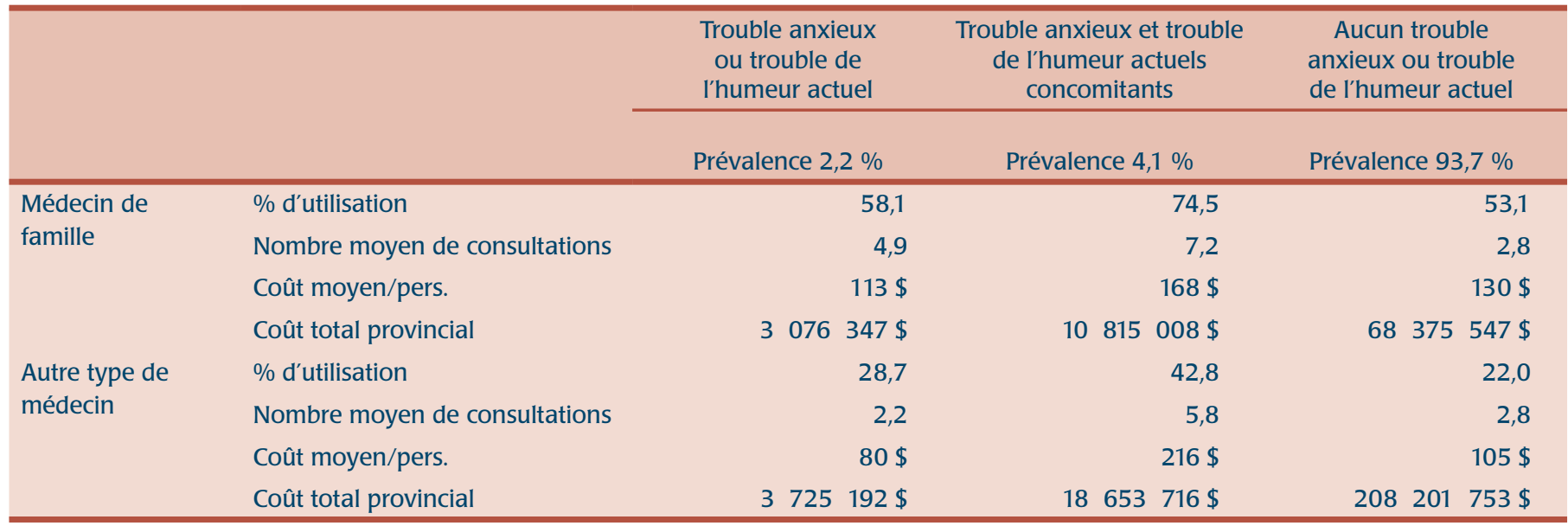


TABLEAU 4

Examens diagnostiques, selon la catégorie de troubles (durant les 6 mois précédant l'enquête)

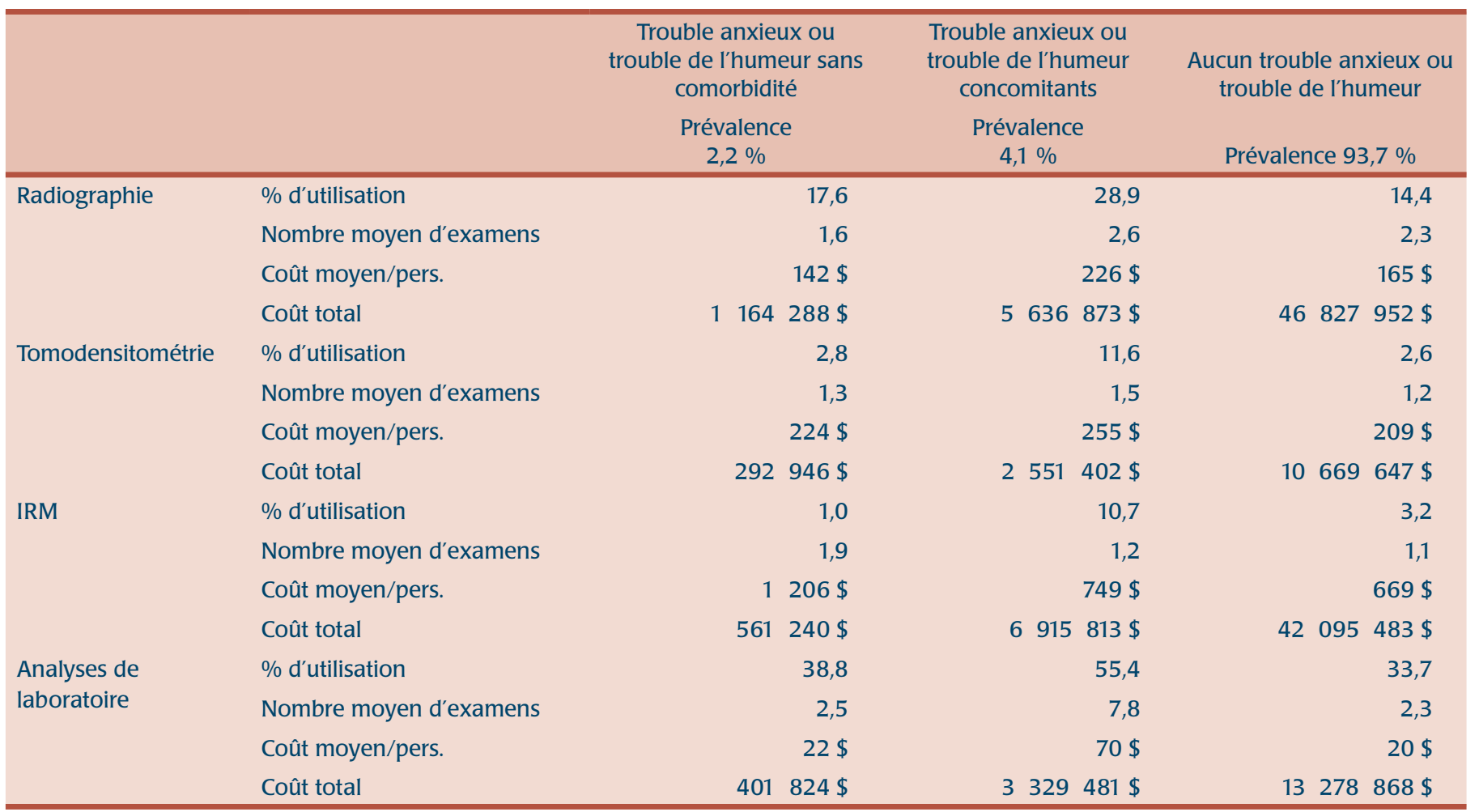

la province atteindrait approximativement Bien que les antidépresseurs et les sédatifs 36 millions de dollars; la présente étude indique, toutefois, que le coût des antidépresseurs en Alberta pourrait être entre 3 et 4 fois supérieur à ce chiffre. L'augmentation des coûts n'est pas inattendue, étant donné que le nombre d'ordonnances de médicaments vendus au détail et le coût moyen par ordonnance sont tous deux en hausse au Canada ${ }^{24}$. hypnotiques aient été les médicaments les plus fréquemment prescrits, un nombre considérable de répondants ont déclaré prendre d'autres médicaments, tels que des antipsychotiques atypiques. Ces médicaments semblent maintenant influer largement sur les coûts totaux des traitements. D’autres analyses doivent être réalisées pour inclure les coûts associés aux anti- psychotiques, aux stimulants et aux psychorégulateurs.

Une autre observation intéressante porte sur la proportion élevée des coûts associés au groupe non atteint d'un trouble. Il faut souligner que la MINI ne permet pas de déceler des troubles traités en voie de rémission, de sorte que, dans une certaine proportion des cas, ces coûts peuvent

TABLEAU 5

Consultations aux urgences et hospitalisations, selon l'état du trouble (durant les 6 mois précédant l'enquête)

\begin{tabular}{|c|c|c|c|c|}
\hline & & $\begin{array}{l}\text { Trouble anxieux ou } \\
\text { trouble de I'humeur sans } \\
\text { comorbidité }\end{array}$ & $\begin{array}{l}\text { Trouble anxieux et } \\
\text { trouble de l'humeur } \\
\text { concomitants }\end{array}$ & $\begin{array}{l}\text { Aucun trouble anxieux } \\
\text { ou trouble de l'humeur }\end{array}$ \\
\hline \multirow{3}{*}{$\begin{array}{l}\text { Consultations aux } \\
\text { urgences }\end{array}$} & $\%$ avec une consultation & 12,3 & 26,9 & 10,7 \\
\hline & Nombre moyen de consultations & 1,3 & 3,1 & 1,5 \\
\hline & Coût total & $351,360 \$$ & $3,348,484 \$$ & $14,209,483 \$$ \\
\hline \multirow[t]{4}{*}{ Hospitalisations } & $\%$ avec hospitalisation & 9,4 & 23,7 & 5,6 \\
\hline & Nombre moyen de jours à l'hôpital & 4,7 & 23,5 & 5,0 \\
\hline & Coût moyen/pers. & $4,988 \$$ & $25,151 \$$ & $5,338 \$$ \\
\hline & Coût total & $21,714,793 \$$ & $514,496,354 \$$ & $587,712,652 \$$ \\
\hline
\end{tabular}


correspondre à une utilisation appropriée des médicaments. Cependant, il est probable qu'une partie de l'utilisation des médicaments dans le groupe non atteint d'un trouble est inappropriée (c.-à-d. absence de trouble indiquant la nécessité d'un traitement). Les symptômes de dépression et d'anxiété peuvent se manifester à la suite de pertes, de menaces ou de facteurs de stress. Le système de santé canadien n'est pas bien structuré pour offrir les courtes interventions psychologiques qui seraient normalement appropriées dans de tels cas. Au Canada, les soins de santé mentale primaires sont habituellement financés selon un modèle d'honoraires à l'acte. Les dispensateurs de soins primaires au Canada rencontrent, en règle générale, un grand nombre de patients durant de courtes consultations. Les antidépresseurs peuvent parfois être prescrits de manière inappropriée à cause du manque de temps ou d'expertise de la part des médecins de premier recours. De même, la prise de sédatifs hypnotiques et d'antipsychotiques atypiques dans le groupe atteint de troubles concomitants pourrait partiellement témoigner de l'accès limité aux traitements non pharmacologiques, tels que les techniques d'hygiène du sommeil et de relaxation. Finalement, une partie de l'utilisation de médicaments dans ce groupe peut être attribuable au traitement de troubles autres que les troubles anxieux et les troubles de l'humeur. Par exemple, les antidépresseurs sont fréquemment prescrits dans les cas de douleurs chroniques et en tant que prophylaxie de la migraine.

Les résultats peuvent avoir des répercussions importantes sur les prochaines études de coûts. Par exemple, l'étude du R.-U. évoquée ci-dessus ${ }^{8}$ a utilisé les données relatives à la facturation pour repérer la "prévalence traitée », mais il se peut que les consultations des sujets recevant un traitement d'entretien au long cours ne soient pas codées expressément pour un trouble anxieux ou un trouble de l'humeur. En fait, toute approche utilisant d'abord les données relatives à la prévalence risque probablement de ne pas tenir compte d'une proportion importante des coûts des médicaments, puisque les troubles traités peuvent être en rémission et qu'ils peuvent aussi ne pas être décelés au moyen des mesures de prévalence fondées sur les symptômes qui sont utilisées dans les enquêtes.

La présente étude comporte plusieurs limites. Tout d'abord, l'étude a mis l'accent sur la prise de psychotropes. Puisque certains troubles médicaux chroniques sont associés aux troubles anxieux et aux troubles de l'humeur, il faut s'attendre à une augmentation des coûts associés aux interventions et aux traitements non psychiatriques chez les sujets présentant des troubles anxieux et des troubles de l'humeur. Nous avons toutefois considéré qu'il était impossible de consigner tous les médicaments pris par les sujets durant l'entrevue téléphonique. Ensuite, la consignation des coûts n'était assurément pas exhaustive. Plus particulièrement, les coûts indirects n’ont pas été évalués. Bien que diverses catégories de coûts aient été estimées chez les répondants présentant ou non des signes d'un trouble, les coûts ne sont pas nécessairement imputables à ces troubles. Par exemple, nous avons examiné les coûts associés aux hospitalisations pour quelque raison que ce soit. Puisque certaines maladies chroniques sont associées à des troubles de l'humeur, les frais hospitaliers peuvent être plus révélateurs de ces troubles médicaux concomitants que la dépression elle-même. Les mécanismes reliant la dépression aux coûts des soins de santé sont vraisemblablement complexes. Par exemple, un effet négatif de la dépression sur l'autotraitement des troubles chroniques peut être la cause d'une plus grande fréquence des admissions à l'hôpital. Il est douteux que ces coûts puissent être attribués en toute confiance à une catégorie diagnostique précise.

D’autres limites sont liées à l'utilisation des méthodes d'enquête téléphonique. La diminution des taux de réponse aux enquêtes téléphoniques au cours des dernières décennies a accru les préoccupations au sujet du fait que de telles études sont sujettes au biais (voir le récent rapport publié par Travaux publics et Services gouvernementaux Canada à l'adresse http://www.pwgsc.gc.ca/por/text/pebptelintro-f.html). Étant donné que la concision est requise durant les entrevues téléphoniques, l'évaluation de l'utilisation a forcément été sommaire. Cependant, la collecte de données par téléphone a bel et bien permis de recueillir des données provenant d'un large échantillon réparti sur un vaste territoire géographique, notamment des renseignements plus détaillés sur l'utilisation de médicaments que ceux habituellement obtenus dans les études de coûts précédentes. Les taux de réponse étaient toutefois moins qu'optimaux, soulevant ainsi des questions au sujet de la validité des estimations. De plus, les intervieweurs ont éprouvé de la difficulté à recueillir de l'information détaillée au sujet des posologies de la part des répondants qui prenaient plusieurs médicaments ou des médicaments selon diverses posologies ou selon des schémas " au besoin ». Étant donné la fréquence élevée des données manquantes, par exemple dans le cas des médicaments autres que les antidépresseurs et les sédatifs hypnotiques, la validité de ces estimations peut être minée.

Il est peu probable que les méthodes d'enquête téléphonique soient suffisantes, en soi, pour constituer une source de données aux fins d'études sur les coûts de la maladie. Cependant, comme le montrent les résultats de cette étude, les données recueillies durant les enquêtes téléphoniques peuvent aider à guider la planification et l'interprétation des études exhaustives sur les coûts de la maladie. La portée de cette étude a été limitée aux coûts directs associés aux troubles anxieux et aux troubles de l'humeur. Une étude détaillée sur les coûts de la maladie devrait également inclure les coûts indirects, tels que la diminution de la productivité attribuable aux conséquences de ces troubles. L'étude actuelle comporte une autre limite, soit l'absence d'évaluation de l'abus d'alcool ou d'autres drogues et de la dépendance à ces substances. Puisque cet aspect n'a pas été mesuré, il a été impossible de déterminer si la fréquence de l'utilisation de sédatifs hypnotiques en particulier peut avoir été lié à l'abus de ces substances ou au dépendance à celles-ci.

Même si de nombreuses estimations de coûts mentionnées dans l'étude actuelle reposaient sur des approximations, ces dernières étaient faites de la même façon dans toutes les catégories de troubles, ce qui devrait faciliter les comparaisons 
valides. Les comparaisons peuvent, toutefois, être trompeuses si la validité des hypothèses employées varie selon les groupes de troubles. Par exemple, si les admissions à l'hôpital pour des troubles anxieux et des troubles de l'humeur coûtent moins cher que les hospitalisations pour d'autres raisons, un biais peut être introduit.

Au Canada, les prochaines études de coûts devront maximiser l'utilisation des données disponibles sur la fréquence du traitement d'entretien et adopter une perspective globale des coûts des médicaments afin d'obtenir des estimations justes des coûts. Les aspects économiques associés aux troubles anxieux et aux troubles de l'humeur semblent évoluer au fil du temps, et les prochaines études de coûts devront tenir compte de ces nouvelles réalités.

\section{Remerciements}

Ce projet a été financé par l'Institut d'économie de la santé dans le cadre de l'Alberta Depression Initiative. Le $\mathrm{D}^{\mathrm{r}}$ Patten est membre de l'Institut d'économie de la santé. Ses travaux sont subventionnés grâce à une bourse nationale de recherche en santé accordée par l'Alberta Heritage Foundation for Medical Research. Les travaux du $\mathrm{D}^{\mathrm{r}}$ Mitton, qui est titulaire de la Chaire de recherche du Canada sur l'élaboration des priorités en matière de soins de santé, sont subventionnés par la Michael Smith Foundation for Health Research.

\section{Références}

1. Insel TR, Fenton WS. Psychiatric epidemiology. It's not just about counting anymore. Arch Gen Psychiatry. 2005;62:590-3.

2. Ayuso-Mateos JL. Global burden of unipolar depressive disorders in the year 2000, Global Burden of Disease Draft 28-05-03, Global Program on Evidence for Health Policy de l'Organisation mondiale de la Santé, 2003.
3. Statistique Canada. Enquête sur la santé dans les collectivités canadiennes : santé mentale et bien-être, Ottawa, Statistique Canada, 9 mars 2004.

4. Patten SB, Wang JL, Williams JVA, Currie SR, Beck CA, Maxwell CJ, et coll. Descriptive epidemiology of major depression in Canada. Can J Psychiatry. 2006;51:84-90.

5. Blazer DG, Kessler RC, McGonagle KA, Swartz MS. The prevalence and distribution of Major Depression in a national community sample: the National Comorbidity Survey. Am J Psychiatry. 1994;151:979-86.

6. Kessler RC, Berglund P, Demler O, Jin R, Koretz D, Merikangas KR, et coll. The epidemiology of major depressive disorder: results from the National Comorbidity Survey Replication (NCS-R). JAMA. 2003; 289:3095-105.

7. Wulsin LR, Vaillant GE, Wells VE. A systematic review of the mortality of depression. Psychosom Med. 1999;61:6-17.

8. Thomas CM, Morris S. Cost of depression among adults in England in 2000. Br J Psychiatry. 2003;183:514-9.

9. Andlin-Sobocki P, Wittchen H-U. Cost of affective disorders in Europe. Eur J Neurol. 2005;12(Suppl. 1):34-8.

10. Kennedy SH, Lam RW, Cohen NL, Ravindran AV, and the CANMAT Depression Work Group. Clinical guidelines for the treatment of depressive disorders. IV. Medications and other biological treatments. Can J Psychiatry. 2001;46(Suppl 1):38S-58S.

11. Stephens T, Dulberg C, Joubert N. La santé mentale de la population canadienne : une analyse exhaustive. Maladies chroniques au Canada. 1999;20: 131-140.
12. Patten SB, Adair CE, Williams JVA, Brant R, Wang JL, Casebeer A et coll. Évaluation de la santé mentale et des maladies mentales par enquête téléphonique : Enquête sur la santé mentale en Alberta. Maladies chroniques au Canada. 2006;27(3): 109-119.

13. Mullet GM. The efficacy of plus-one dialing: Self reported status. American Statistical Association: Proceedings of the Section on Survey Research Methods 1982;575-6.

14. Potthoff RF. Telephone sampling in epidemiological research: to reap the benefits, avoid the pitfalls. Am J Epidemiol. 1994; 139:967-78.

15. Lecubier Y, Sheehan DV, Weiller E, Amorim P, Bonora I, Sheehan KH, et coll. The MINI International Neuropsychiatric Interview (MINI). A short diagnostic structured interview: reliability and validity according to the CIDI. Eur Psychiatry. 1997;12:224-31.

16. Sheehan DV, Lecubier Y, Sheehan $\mathrm{KH}$, Janavs J, Weiller E, Keskiner A, et coll. The validity of the Mini International Neuropsychiatric Interview (MINI) according to the SCID-P and its validity. Eur Psychiatry. 1997;12:232-41.

17. Narrow WE, Rae DS, Robins LN, Regier DA. Revised prevalence estimates of mental disorders in the United States: using a clinical significance criterion to reconcile 2 surveys' estimates. Arch Gen Psychiatry. 2002;59:115-23.

18. Waraich PS, Goldner EM, Somers JM, Hsu L. Prevalence and incidence studies of mood disorders: a systematic review of the literature. Can J Psychiatry. 2004;49:124-38.

19. Kessler RC, Chiu WT, Demler O, Merikangas KR, Walters EE. Prevalence, severity, and comorbidity of 12-month DSM-IV disorders in the National Comorbidity Survey Replication. Arch Gen Psychiatry. 2005 Jun; 62(6):617-27. 
20. Statistique Canada. Enquête sur la santé dans les collectivités canadiennes : santé mentale et bien-être, Le Quotidien, le mercredi 3 septembre 2003. URL : http:// www.statcan.ca/Daily/Français/030903/ 9030903a.htm

21. Patten SB, Beck CA, Kassam A, Williams JVA, Barbui C, Metz LM. Long-term medical conditions and major depression: strength of association in the general population. Can J Psychiatry. 2005;50:195-202.
22. Gagnon LM, Patten SB. Major depression and its association with long-term medical conditions. Can J Psychiatry. 2002;47:167-73.

23. Wells KB, Golding JM, Burnam MA. Psychiatric disorder in a sample of the general population with and without chronic medical conditions. Am J Psychiatry. 1988;145(8):976-81.
24. Pharmaceutical trends: Top products, classes and companies sourced from IMS data. URL : http://www.imshealthcanada. com/web/channel/0,3147,77303623_6387 2702_77770072,00.html 